\title{
Frequency of colistin and fosfomycin resistance in carbapenem-resistant Enterobacteriaceae from a tertiary care hospital in Karachi
}

\section{Salima Qamar \\ Najma Shaheen \\ Sadia Shakoor \\ Joveria Farooqi \\ Kauser Jabeen \\ Rumina Hasan}

Clinical Microbiology, Department of Pathology And Laboratory Medicine, Aga Khan University Hospital, Karachi, Pakistan

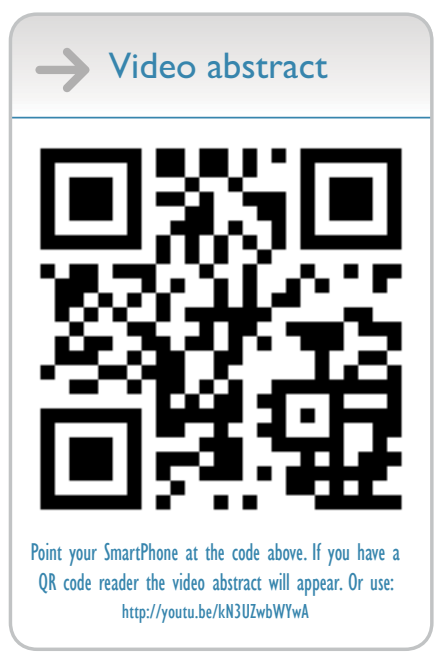

Correspondence: Rumina Hasan Aga Khan University Hospital, Section Microbiology, Ist floor, Supariwala Building, P.O. Box 3500, Karachi, Pakistan Tel +92 2l 3486 I64I

Email rumina.hasan@aku.edu
This article was published in the following Dove Press journal:

Infection and Drug Resistance

31 July 2017

Number of times this article has been viewed

Introduction: Management of infections with carbapenem-resistant Enterobacteriaceae (CRE) is challenging. In recent times, agents such as colistin and fosfomycin have been used in combination with other antibiotics to treat such infections. In this study, we aim to seek frequency of colistin and fosfomycin resistance in CRE from Pakistan.

Methods: This study was conducted at clinical laboratories, Aga Khan University Hospital. In total, $251 \mathrm{CRE}$ were included in the study. Colistin minimum inhibitory concentrations (MICs) were performed using broth microdilution (BMD) method and VITEK ${ }^{\circledR} 2$ system, whereas fosfomycin susceptibility was performed using Kirby-Bauer method. $\mathrm{MIC}_{50}$ and $\mathrm{MIC}_{90}$ were calculated for colistin and agreement between VITEK and BMD was also calculated.

Results: Out of 251 strains colistin MIC of $\geq 4 \mu \mathrm{g} / \mathrm{mL}$ was seen in 40 (15.9\%). Of these strains $20(50 \%)$ were Klebsiella pneumoniae. Colistin $\mathrm{MIC}_{50}$ and $\mathrm{MIC}_{90}$ were found to be 0.5 and $16 \mu \mathrm{g} / \mathrm{mL}$, respectively. BMD and VITEK 2 showed 100\% categorical agreement. Essential agreement was $88.5 \%$ with kappa score 0.733 indicating strong agreement between VITEK and BMD. 31 out of 251 (12.3\%) CREs were resistant to fosfomycin.

Conclusion: Study shows frequency of colistin and fosfomycin resistance to be $15.9 \%$ and $12.3 \%$, respectively. In countries where rate of CREs is high, emerging resistance against these last resort antibiotics is alarming as it leaves clinicians with almost no options to manage such multidrug resistant and extensively drug resistant infections.

Keywords: emerging drug resistance, colistin resistance, fosfomycin resistance, carbapenam resistant enterobacteriaceae, salvage antibiotics

\section{Introduction}

Carbapenem-resistant Enterobacteriaceae (CRE) are increasingly being identified worldwide and pose a major threat to the treatment of infectious diseases and to infection control within heath care facilities. ${ }^{1}$ In 2014, a meta-analysis showed mortality rates attributable to CRE to be $26 \%-44 \%$. $^{2}$ Frequency of CRE is increasing in worldwide..$^{3,4}$ In Pakistan, the rates have been increasing steadily, data from our hospital showed increase in carbapenem-resistant Escherichia coli, Enterobacter spp. and Klebsiella pneumoniae from 5\%, 7\% and $15 \%$ in 2013 to $6 \%, 23 \%$ and $22 \%$ by 2015 , respectively. ${ }^{5}$

With increasing antimicrobial resistance and given the paucity of new drugs, the focus of management has shifted to older agents. ${ }^{6}$ Currently, such antibiotics in use in Pakistan include colistin and fosfomycin. It has earlier been shown that treatment of CRE with combination of carbapenem and colistin is associated with better outcomes $^{7}$ leading to increased use of colistin in clinical practice. ${ }^{8}$ Fosfomycin has traditionally been used to treat urinary tract and gastrointestinal infections. A meta- 
analysis including 62 studies, showed utility of fosfomycin either alone or in combination with other antibiotics for the treatment of pneumonia, osteomyelitis, meningitis, ear, nose, throat infections and gynecological infections, ${ }^{9}$ hence, identifying fosfomycin as having potential for treatment of invasive infections. In February 2010, intravenous (IV) fosfomycin was prospectively evaluated for the treatment of carbapenem-resistant $K$. pneumoniae wherein all patients had bacteriological clearance with no adverse reactions. ${ }^{10}$

Given rising antimicrobial resistance and reports of CRE, this study was designed to evaluate the frequency of colistin and fosfomycin resistance in carbapenem-resistant Gramnegative bacterial infections in our population.

\section{Materials and methods Study setting}

This was a prospective study conducted on archived bacterial isolates from clinical specimens received in the Clinical Microbiology Laboratory, Aga Khan University. The archived strains were stored at $-80^{\circ} \mathrm{C}$ in glycerol phosphate broth and were revived on MacConkey agar before use.

\section{Bacterial isolates}

All CRE isolated from January 2015 to August 2016 were included. CREs were defined using CLSI 2015 cut-offs for ertapenem, imipenem or meropenem. ${ }^{11}$ Identification of strains included in the study was done using API 20E system. Strains with inherent resistance to colistin such as those belonging to genus Proteus, Providencia and Serratia were excluded from the study.

- Susceptibility testing: Carbapenem susceptibilities were performed by disc diffusion (Kirby-Bauer) method on Mueller Hinton agar ${ }^{11}$ and by VITEK 2 system. ${ }^{12}$ Isolates were labeled as CREs by disc diffusion zone diameters and by VITEK minimum inhibitory concentrations (MICs). For interpretation CLSI 2015 breakpoints for imipenem, ertapenem and meropenem, that is, for ertapenem disc diameter $\leq 19 \mathrm{~mm}$ or $\mathrm{MIC} \geq 1$, for imipenem and meropenem disc diameter $\leq 21 \mathrm{~mm}$ or $\mathrm{MIC} \geq 2$ were used. ${ }^{11}$

- Colistin susceptibility was performed as follows: broth microdilution using 96-well microtiter plates to perform MICs using cation adjusted Mueller Hinton broth as culture medium. Drug concentration ranging from 0.03 to $16 \mu \mathrm{g} / \mathrm{mL}$ was used. MICs were performed in batches of 10-20 strains per batch, 5 days a week from July 2016 till August 2016 on archived strains. Colistin sulfate powder was obtained from Sigma-Aldrich Co. (St Louis,
MO, USA). ATCC E. coli 25922 and ATCC P. aeruginosa 27853 were used as quality control strains. Results were read at 24 hours and interpreted using colistin cut-offs for Enterobacteriaceae in EUCAST 2016, that is, MIC $\leq 2$ as sensitive. ${ }^{13}$ Colistin MICs were also determined using automated VITEK 2 system. Results were compared with broth microdilution. Broth microdilution was used as the gold standard. Agreement between broth and VITEK was calculated.

- Fosfomycin susceptibility was tested by disc diffusion method using fosfomycin trometamol disc $(200 \mu \mathrm{g})$ (Thermo Fisher Scientific, Waltham, MA, USA) containing $50 \mu \mathrm{g}$ G6PD on Muller Hinton agar. Results were interpreted using CLSI 2015 disc diffusion cut-offs for $E$. coli in urinary tract isolates, that is, $\geq 16$ as sensitive, $13-15$ intermediate and $\leq 12$ as resistant. ${ }^{11}$

\section{Ethical approval}

As this study was conducted on archived bacterial isolates and not on patients' samples, it was given exemption by the ethical review committee Aga Khan University Hospital (ERC\# 3992-PAT-ERC-16).

\section{Statistical analysis}

Data entered in Microsoft Excel $2010^{\circledR}$ and imported to Stata $\mathrm{SE}$ (version 12) was used to calculate $\mathrm{MIC}_{50}$ and $\mathrm{MIC}_{90}$. $\mathrm{MIC}_{50}$ and $\mathrm{MIC}_{90}$ were defined as the lowest concentration of the antibiotic at which $50 \%$ and $90 \%$ of the tested strains were inhibited. Categorical and essential agreement between VITEK and broth microdilution was calculated using Kappa scores.

\section{Results}

The study included 251 strains. These included E. coli $39.04 \%$ (98/251) followed by K. pneumoniae 31\% (78/251), Raoultella spp. 15.5\% (Raoultella terrigena and Raoultella ornithinolytica) (39/251), Enterobacter spp. 7.5\% (19/251), Klebsiella oxytoca 5.1\% (13/251) and Citrobacter spp. $1.1 \%(3 / 251)$. Details regarding source and characteristics of patients from whom these isolates were obtained are provided in Table $\mathrm{S} 1$.

\section{$\mathrm{MIC}_{50}$ and $\mathrm{MIC}_{90}$}

Colistin MICs by broth microdilution was performed on all strains. $\mathrm{MIC}_{50}$ value was calculated to be $0.5 \mu \mathrm{g} / \mathrm{mL}$, whereas $\mathrm{MIC}_{90}$ was $16 \mu \mathrm{g} / \mathrm{mL}$. $\mathrm{MIC}_{50}$ and $\mathrm{MIC}_{90}$ values among different bacterial species was separately calculated (Figure 1).

Colistin MICs was $\geq 4 \mu \mathrm{g} / \mathrm{mL}$ in 40 out of 251 (15.9\%) study strains which were identified as being colistin resistant. 


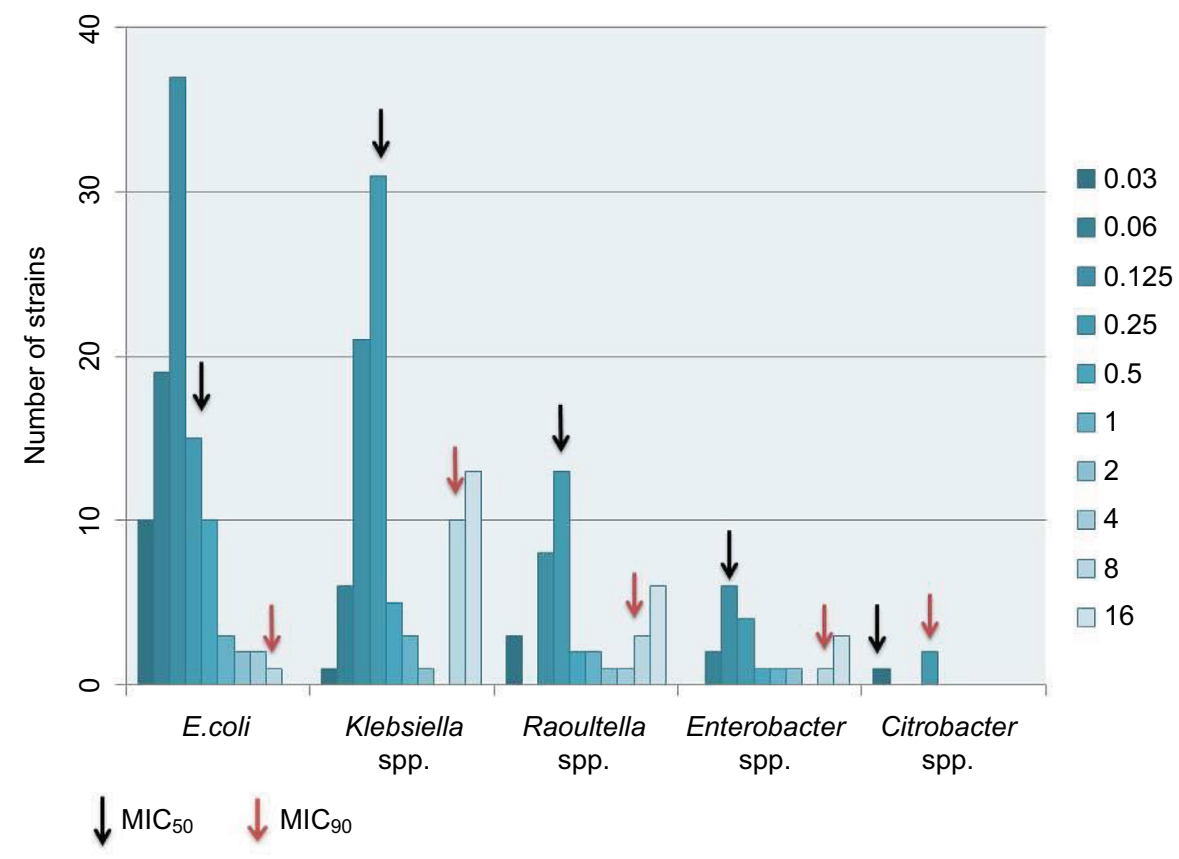

Figure I Groups of Enterobacteriaceae with MIC ranges from 0.03 to $16 \mu \mathrm{g} / \mathrm{mL}$.

Out of these 40 strains $50 \%$ were K. pneumonia (n: 20), $22.5 \% R$. terrigena (n:10), 10\% Enterobacter spp. (n:4), $7.5 \%$ E. coli (n:3) and $7.5 \%$ K. oxytoca $(\mathrm{n}: 3)$.

\section{Agreement between VITEK and broth}

VITEK MICs were available for 149 strains and agreement was calculated. Broth and VITEK showed $100 \%$ categorical agreement with kappa score of 1, CI (0.84-1.16). Essential agreement was calculated to be $88.59 \%$ with kappa score of 0.7334, CI (0.6-0.798).

\section{Fosfomycin susceptibility}

Among this CRE collection, rate of fosfomycin resistance was $12.3 \%$ (31/251). In the colistin-resistant CRE subgroup (n:40) 35 out of 40 remained susceptible to fosfomycin while 5 stains were found resistant to both colistin and fosfomycin simultaneously.

\section{Discussion}

Multidrug resistant (MDR) and extensively drug resistant (XDR) bacteria are a global threat. In countries with limited resources, over-stretched health systems make it difficult to manage such infections. Furthermore, inadequate implementation of infection control policies contribute toward dissemination of both nosocomial infections as well as of antimicrobial resistance.
Our study showed $15.9 \%$ colistin and $12.3 \%$ fosfomycin resistance among CREs. Both colistin and fosfomycin are salvage antibiotics for treating MDR and XDR infections. Hence emerging resistance against these agents significantly reduces available treatment options. Despite their potential side effects and high cost, in the absence of alternatives, clinicians have had no option but to use these antibiotics.

Aga Khan University hospital is a 680-bedded tertiary care hospital which caters to thousands of patients all year round. Our laboratory has more than 200 collection units and participates in external quality assurance surveys from College of American Pathologist on regular basis. Since rate of CRE in the hospital has been increasing over time, colistin or fosfomycin are only prescribed for patients on clinician's advice and their use is closely monitored by the pharmacy service. As per Antibiotic policy of the hospital, IV fosfomycin and colistin have been labeled as restricted and controlled antibiotics which require mandatory approval from the infectious diseases pharmacist or ID physician for dispense.

Colistin resistance among CRE has been previously reported. Most of these studies have been case reports or outbreaks in hospital settings. An outbreak in three institutions in Detroit, MI, USA reported a cluster of colistin-resistant, carbapenem-resistant $K$. pneumoniae infection in 2009. ${ }^{14}$ Another study from Italy also reported emergence of colistin resistance in carbapenem-resistant $K$. pneumoniae strains 
collected from 21 hospital laboratories during November 2013 to April 2014. ${ }^{15}$ Goel et al reported emergence of a cluster of 24 cases of colistin resistant $K$. pneumoniae in oncology unit in India. ${ }^{16}$

Earlier, colistin resistance was known to be mediated by chromosomal mutations which altered the lipopolysaccharide of the bacterial outer membrane. These include $\operatorname{mgr} B$, phoP/phoQ, pmrA, pmrB, $p m r C$, and $\operatorname{crr} A B C .{ }^{17}$ In 2015, a study from China reported emergence of plasmid mediated colistin resistance ( $m c r-1)$ in $E$. coli and $K$. pneumoniae from human and animals. ${ }^{18} \mathrm{~A}$ variant of $m c r-1$ ( $\left.m c r-1.2\right)$ and $m c r-2$ were also detected in Italy and Belgium. ${ }^{19,20}$ Rapid rise of resistance in such a brief period is alarming and poses a major threat in countries with high CRE prevalence such as Pakistan, further minimizing available antibiotic options for treatment of CRE infections.

Due to excessive and inappropriate use of colistin, resistance has not only emerged among CRE but other bacterial species as well. In 2002, an outbreak of colistin-resistant Pseudomonas aeruginosa in a pulmonology unit in the UK created an infection control concern, ${ }^{21}$ over the years this was followed with different reports from Australia, India and Iran where colistin resistance was documented in Acinetobacter baumanii as well. ${ }^{22-24}$

With limited available options for treating CRE infections, fosfomycin held promise. An earlier study on $152 \mathrm{MDR}$ (including 85 XDR) Enterobacteriaceae isolates, reported overall susceptibility against fosfomycin of $92.8 \%(141 / 152) .{ }^{25}$ These findings concurred with another study published in the same year, 2009, reporting 7\% fosfomycin resistance in prospectively collected $68 \mathrm{~K}$. pneumoniae isolates of which 23 were also nonsusceptible to tigecycline and/or colistin. ${ }^{26} \mathrm{~A}$ recent study from Germany in 2013 tested fosfomycin against CRE and reported $78 \%$ of the tested strain as fosfomycin sensitive. ${ }^{27}$ Hence, our data reporting $12.3 \%$ resistance in CRE suggests increasing resistance against this antibiotic.

Our study has few limitations. Due to financial constrain test strains were only identified on basis of biochemical identification using API 20E. Neither molecular identification of the isolates, nor resistance gene detection was performed for mcr- $1, \operatorname{mrgB}$ or other mutations. Hence additional studies are required to identify genetic mutations associated with colistin resistance in this part of the world. As we tested colistin and fosfomycin using two different methods (broth microdilution and disc diffusion) we could not look for synergism and, therefore, further studies are needed to evaluate synergy between different antibiotics combination for the treatment of CRE.

\section{Conclusion}

Our data suggest emerging in vitro resistance against colistin and fosfomycin in CREs from Pakistan. Our findings suggest a need for regular monitoring of antimicrobial resistance in the country including for colistin and fosfomycin. Our data further highlights the urgency for developing and implementing a national policy toward control of antimicrobial resistance in Pakistan.

\section{Disclosure}

The authors report no conflicts of interest in this work.

\section{References}

1. Nordmann P, Naas T, Poirel L. Global spread of Carbapenemase producing Enterobacteriaceae. Emerg Infect Dis. 2011;17(10):1791-1798.

2. Falagas ME, Tansarli GS, Karageorgopoulos DE, Vardakas KZ. Deaths attributable to carbapenem-resistant Enterobacteriaceae infections. Emerg Infect Dis. 2014;20(7):1170-1175.

3. Kumarasamy KK, Toleman MA, Walsh TR, et al. Emergence of a new antibiotic resistance mechanism in India, Pakistan, and the UK: a molecular, biological, and epidemiological study. Lancet Infect Dis. 2010;10(9):597-602.

4. Gupta N, Limbago BM, Patel JB, Kallen AJ. Carbapenem-resistant enterobacteriaceae: epidemiology and prevention. Clin Infect Dis. 2011;53(1): 60-67.

5. Pakistan antimicrobial resistance network, 2016. Available from http:// www.parn.org.pk/index_files/Antimicrobial $\% 20$ data.html. Accessed February 10, 2017.

6. Falagas ME, Grammatikos AP, Michalopoulos A. Potential of oldgeneration antibiotics to address current need for new antibiotics. Expert Rev Anti Infect Ther. 2008;6(5):593-600.

7. Van Duin D, Kaye KS, Neuner EA, Bonomo RA. Carbapenem-resistant Enterobacteriaceae: a review of treatment and outcomes. Diagn Microbiol Infect Dis. 2013;75(2):115-120.

8. Hanulík V, Suchánková H, Urbánek K, et al. [Effect of colistin consumption and prevalence of colistin-resistant bacteria]. Klin Mikrobiol Infekc. 2013;19(2):52-55. Czech with English Abstract.

9. Falagas ME, Giannopoulou KP, Kokolakis GN, Rafailidis PI. Fosfomycin: use beyond urinary tract and gastrointestinal infections. Clin Infect Dis. 2008;46(7):1069-1077.

10. Livermore DM, Warner M, Mushtaq S, Doumith M, Zhang J, Woodford $\mathrm{N}$. What remains against carbapenem-resistant Enterobacteriaceae? Evaluation of chloramphenicol, ciprofloxacin, colistin, fosfomycin, minocycline, nitrofurantoin, temocillin and tigecycline. Int JAntimicrob Agents. 2011;37(5):415-419.

11. CLSI. Performance standards for Antimicrobial susceptibility testing. Approved Guideline. 2014;34(1):M100-S24.

12. Ling TK, Tam PC, Liu ZK, Cheng AF. Evaluation of VITEK 2 rapid identification and susceptibility testing system against gram-negative clinical isolates. J Clin Microbiol. 2001;39(8):2964-2966.

13. European committee of antimicrobial susceptibility testing (EUCAST) 2016. Available from: http://www.eucast.org/clinical_breakpoints. Accessed october 22, 2016.

14. Marchaim D, Chopra T, Pogue JM, et al. Outbreak of colistin-resistant, carbapenem-resistant Klebsiella pneumoniae in metropolitan Detroit, Michigan. Antimicrob Agents Chemother. 2011;55(2):593-599.

15. Monaco M, Giani T, Raffone M, et al. Colistin resistance superimposed to endemic carbapenem-resistant Klebsiella pneumoniae: a rapidly evolving problem in Italy, November 2013 to April 2014. Euro Surveill. 2014;19(42):pii20939. 
16. Goel G, Hmar L, De MS, Bhattacharya S, Chandy M. Colistin-resistant Klebsiella pneumoniae: report of a cluster of 24 cases from a new oncology center in eastern India. Infect Control Hosp Epidemiol. 2014;35(08):1076-1077.

17. Cannatelli A, D’Andrea MM, Giani T, et al. In vivo emergence of colistin resistance in Klebsiella pneumoniae producing KPC-type carbapenemases mediated by insertional inactivation of the $\mathrm{PhoQ} / \mathrm{PhoP}$ mgrB regulator. Antimicrob Agents Chemother. 2013;57(11):5521-5526.

18. Liu YY, Wang Y, Walsh TR, et al. Emergence of plasmid-mediated colistin resistance mechanism MCR-1 in animals and human beings in China: a microbiological and molecular biological study. Lancet Infect Dis. 2016;16(2):161-168.

19. Di Pilato V, Arena F, Tascini C, et al. mcr-1.2, a new mcr variant carried on a transferable plasmid from a colistin-resistant KPC carbapenemaseproducing Klebsiella pneumoniae strain of sequence type 512. Antimicrob Agents Chemother. 2016;60(9):5612-5615.

20. Xavier BB, Lammens C, Ruhal R, et al. Identification of a novel plasmidmediated colistin-resistance gene, mcr-2, in Escherichia coli, Belgium, June 2016. Euro Surveill. 2016;21(27).

21. Denton M, Kerr K, Mooney L, et al. Transmission of colistin-resistant Pseudomonas aeruginosa between patients attending a pediatric cystic fibrosis center. Pediatr Pulmonol. 2002;34(4):257-261.
22. Li J, Rayner CR, Nation RL, et al. Heteroresistance to colistin in multidrug-resistant Acinetobacter baumannii. Antimicrob Agents Chemother. 2006;50(9):2946-2950.

23. Taneja N, Singh G, Singh M, Sharma M. Emergence of tigecycline \& colistin resistant Acinetobacter baumanii in patients with complicated urinary tract infections in north India. Indian JMed Res. 2011;133(6):681.

24. Bahador A, Taheri M, Pourakbari B, et al. Emergence of rifampicin, tigecycline, and colistin-resistant Acinetobacter baumannii in Iran; spreading of MDR strains of novel International Clone variants. Microb Drug Resist. 2013;19(5):397-406.

25. Falagas ME, Maraki S, Karageorgopoulos DE, Kastoris AC, Mavromanolakis E, Samonis G. Antimicrobial susceptibility of multidrug-resistant (MDR) and extensively drug-resistant (XDR) Enterobacteriaceae isolates to fosfomycin. Int $J$ Antimicrob Agents. 2010;35(3):240-243.

26. Endimiani A, Patel G, Hujer KM, et al. In vitro activity of fosfomycin against blaKPC-containing Klebsiella pneumoniae isolates, including those nonsusceptible to tigecycline and/or colistin. Antimicrob Agents Chemother. 2010;54(1):526-529.

27. Kaase M, Szabados F, Anders A, Gatermann SG. Fosfomycin susceptibility in carbapenem-resistant Enterobacteriaceae from Germany. J Clin Microbiol. 2014;52(6):1893-1897. 


\section{Supplementary material}

Table SI General characteristics of the bacterial isolates included in the study

\begin{tabular}{ll}
\hline General characteristics of the study & $\mathbf{N}=\mathbf{2 5} \mathbf{~}$ \\
\hline Mean, age & 50.9 years \\
Gender & \\
Male (\%) & 56.8 \\
Female (\%) & 43.13 \\
Site of organism recovery & \\
Urine & 143 \\
Blood & $6 \mathrm{I}$ \\
Cerebrospinal fluid & 4 \\
Pus & $\mathrm{II}$ \\
Tissue & 7 \\
Respiratory specimen & 14 \\
Other body fluids & $\mathrm{II}$ \\
\hline
\end{tabular}

Infection and Drug Resistance is an international, peer-reviewed openaccess journal that focuses on the optimal treatment of infection (bacterial, fungal and viral) and the development and institution of preventive strategies to minimize the development and spread of resistance. The journal is specifically concerned with the epidemiology of antibiotic resistance and the mechanisms of resistance development and diffusion in both hospitals and the community. The manuscript management system is completely online and includes a very quick and fair peerreview system, which is all easy to use. Visit http://www.dovepress.com/ testimonials.php to read real quotes from published authors.

Submit your manuscript here: https://www.dovepress.com/infection-and-drug-resistance-journal 